\title{
Automatic 3D Segmentation of the Kidney in MR Images Using Wavelet Feature Extraction and Probability Shape Model
}

\author{
Hamed Akbari ${ }^{a}$ and Baowei Fei ${ }^{a, b, *}$ \\ ${ }^{\mathrm{a}}$ Department of Radiology and Imaging Sciences, ${ }^{\mathrm{b}}$ Department of Biomedical Engineering, \\ Emory University and Georgia Institute of Technology, Atlanta, GA \\ *E-mail: bfei@emory.edu, Website: http://feilab.org
}

\begin{abstract}
Numerical estimation of the size of the kidney is useful in evaluating conditions of the kidney, especially, when serial MR imaging is performed to evaluate the kidney function. This paper presents a new method for automatic segmentation of the kidney in three-dimensional (3D) MR images, by extracting texture features and statistical matching of geometrical shape of the kidney. A set of Wavelet-based support vector machines (W-SVMs) is trained on the MR images. The W-SVMs capture texture priors of MRI for classification of the kidney and non-kidney tissues in different zones around the kidney boundary. In the segmentation procedure, these W-SVMs are trained to tentatively label each voxel around the kidney model as a kidney or non-kidney voxel by texture matching. A probability kidney model is created using 10 segmented MRI data. The model is initially localized based on the intensity profiles in three directions. The weight functions are defined for each labeled voxel for each Wavelet-based, intensity-based, and model-based label. Consequently, each voxel has three labels and three weights for the Wavelet feature, intensity, and probability model. Using a 3D edge detection method, the model is re-localized and the segmented kidney is modified based on a region growing method in the model region. The probability model is re-localized based on the results and this loop continues until the segmentation converges. Experimental results with mouse MRI data show the good performance of the proposed method in segmenting the kidney in MR images.
\end{abstract}

Keywords: Kidney, image segmentation, Wavelet, Support Vector Machines, MRI, Probability Shape Model, Polycystic kidney disease

\section{INTRODUCTION}

Polycystic kidney disease (PKD) is a genetic disease that passed down through families, usually as an autosomal dominant trait. In early stages of the disease, the cysts cause the kidney swelling, disrupting kidney function and leading to chronic high blood pressure and kidney infections. This disease leads to enlarged bilateral kidneys with multiple cysts. The severity of the renal functional impairment is correlated with the size of the kidneys. Usually a larger kidney accompanies with more sever renal failure [1-4, 5 ]. Serial MRI evaluations can provide high-resolution anatomic structure of the kidneys and thus could be a useful tool for the assessment of different treatments [6-9].

In this paper, a new automatic segmentation method is proposed and evaluated to segment the kidneys in MR images that may be utilized for noninvasive monitoring and assessment of the kidneys. A variety of methods for semiautomatic or automatic segmentation of kidney MR images have been proposed. Our group previously proposed three approaches to segment the kidney in MR images [8-10]. A semiautomatic segmentation method was proposed, where the average of the manually segmented boundaries from different images was used as an initial model for automatic segmentation [9]. To segment a new image, a point was manually placed within the kidney and the model was then deformed to the renal boundary using spline curves by optimizing the intensity gradients along the boundary. A minimal path segmentation method was developed for segmenting mouse kidneys in MR images [8]. A dynamic programming and a minimal path segmentation approach was used to detect the optimal path within a weighted graph between two end points. In this minimal path method, the energy function combines distance and gradient information to guide the marching curve and thus to evaluate the best path and to span a broken edge. Initial end points were placed automatically and dynamic programming was used to optimize and update end points during the searching procedure. Principle component analysis (PCA) was used to generate a deformable model, which serves as the prior knowledge for the selection of initial end points and for the evaluation of the best path [8]. In this paper, we propose a 3D method for automatically segmenting the kidney in 3D MR images using Wavelet features and kidney geometry.

Medical Imaging 2012: Image Processing, edited by David R. Haynor, Sébastien Ourselin,

Proc. of SPIE Vol. 8314, 83143D - (C) 2012 SPIE · CCC code: 0277-786X/12/\$18 - doi: 10.1117/12.912028 


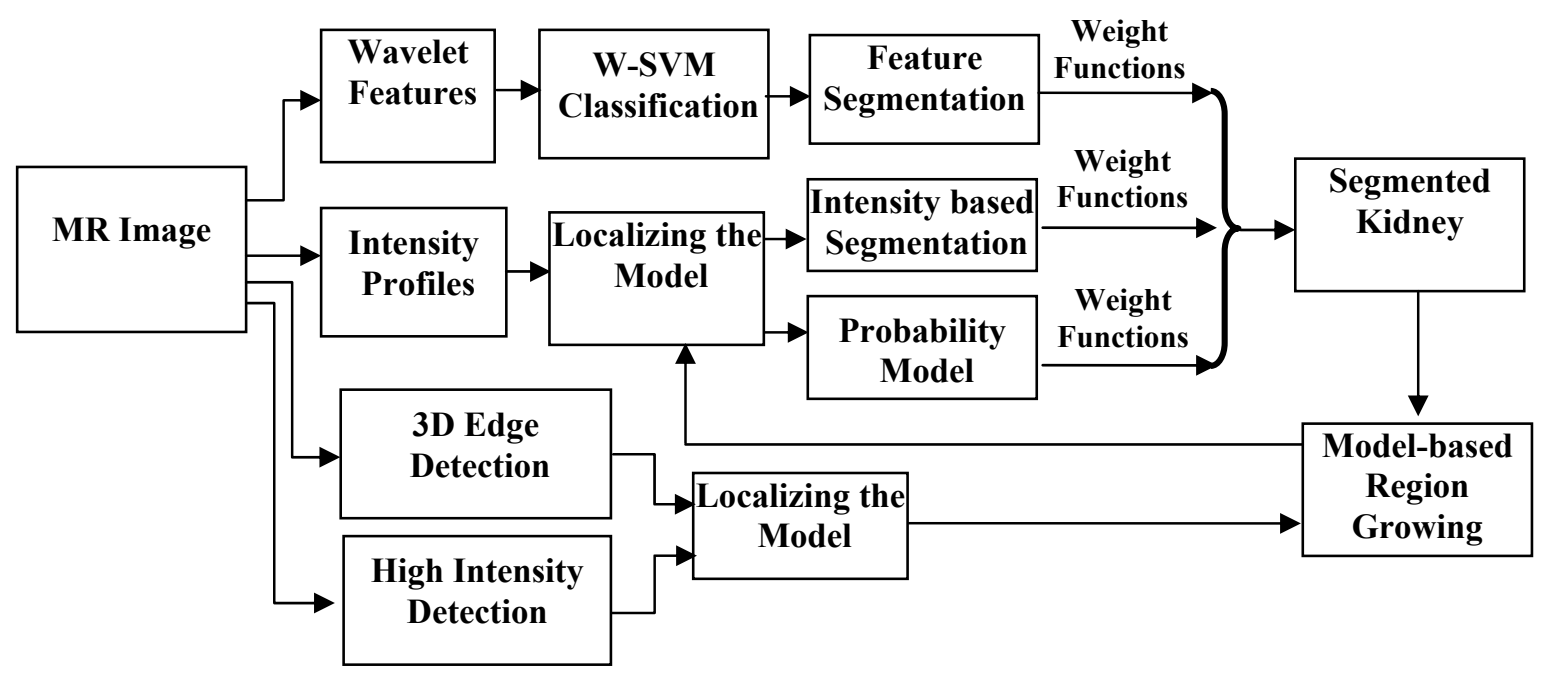

Figure 1. Flow chart of the segmentation method.

\section{METHODS}

This method is based on our segmentation method for prostate ultrasound images [11]. The proposed method in this paper consists of the training and application stages. Figure 1 shows the flowchart of the method. Ten training MR images were employed to train the wavelet feature classification and to make a predefined model. The kidney boundaries have been manually defined.

A kidney shape model is created based on the allowable models of shape variations and its probability. This model is employed to modify the kidney boundaries. The kidney textures are captured by training the Wavelet-based support vector machines (W-SVMs). With integrating texture features and geometrical data, W-SVMs can robustly differentiate the kidney tissue from the adjacent tissues. The trained W-SVMs are employed to tentatively label the respective voxels around the surface into kidney and non-kidney tissue based on their texture features from different Wavelet filters. Subsequently, after the translation of the shape model to the kidney region that are defined by intensity profiles of the MRI, the surface of the kidney is driven to the boundary between the tentatively labeled kidney and non-kidney tissues based on defined weighting functions and labeled voxels. The intensity profiles is calculated using the intensities of the cube passes the center of mass of the MR images in sagittal, coronal, and transverse planes. Figure 2 shows the intensity profiles of MR data from in five mice. A 3D edge detection method is employed based on Canny edge detection. The high-intensity regions in the MR images are combined with the detected edge. The combined image is used to localize the model using the lower edge of the kidney. The segmented kidney is modified based on a region growing method in the model-defined region. The model is then re-localized based on the new detected region. 

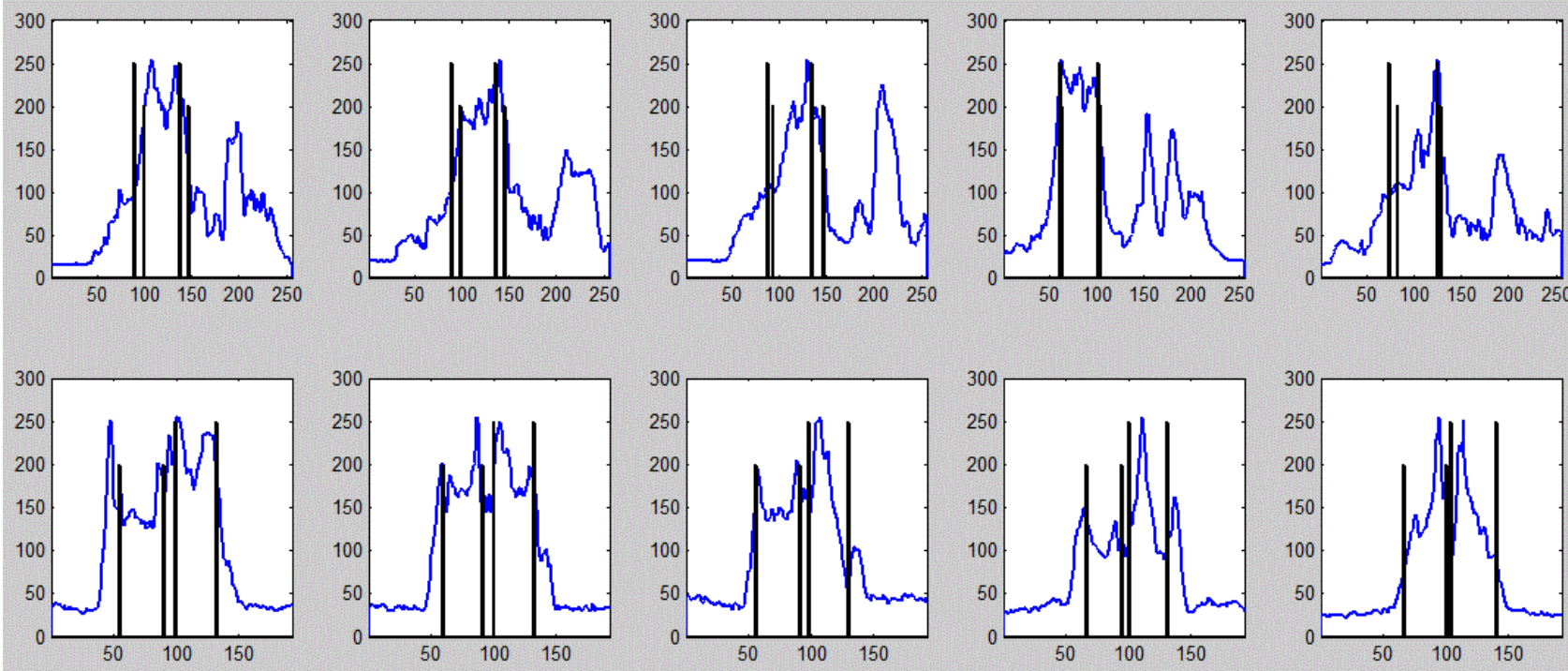

Figure 2. The intensity profiles of MR data from five mice. The intensity profile is shown in blue lines. The locations of the kidney boundaries are shown in black, vertical lines. Top: The X-axis from left to right represents the tail-to-head direction of the mouse. Bottom: The X-axis represents the left-to-right of the mouse. The vertical lines with different heights represent the two kidneys.

\subsection{W-SVMs}

MR image textures can provide important features for accurately defining the kidney, especially for the regions where kidney boundaries are not clear. Daubechies 2, Symlets 2, Symlets 2, Coiflets 1, Biorthogonal 1.3 and 1.5 are employed to extract the texture features of the kidney. Designing biorthogonal wavelets allows more degrees of freedom comparing to orthogonal wavelets. One additional degree of freedom is the possibility to construct symmetric wavelet functions. Using different types of Wavelet can detect different texture properties of the kidney. The W-SVMs are trained to adaptively label the tissue based on its texture and location. The W-SVM is composed of six wavelet filter banks, voxel coordinates, and a Kernel Support Vector Machine (K-SVM). Figure 3 shows 6 wavelet filters that were employed for feature extraction. The wavelet filters are employed to extract texture features from MR images, and the K-SVM is used to nonlinearly classify the Wavelet texture features for tissue differentiation. The trained W-SVMs are employed to tentatively label each voxel into kidney and non-kidney tissues in the application stage.

\subsection{Probability model}

A kidney probability model was used for modifying the segmentation. In this study, we registered ten segmented kidneys using an affine transformation. Other registration methods can also be used to create the model [12-17]. The registration approach that employed for creating the model is based on the principal axis transformation. This method was chosen because of its computational properties, speed and simplicity. The kidney volume was translated and rotated with respect to each other. The principal axis transformation is known from the classical theory of rigid bodies. A rigid body is uniquely located by knowledge of the position of its center of mass and its orientation (rotation) with respect to its center of mass. The center of mass, inertia matrix, and principal axes can be determined for any rigid body. For simple geometric shapes, the principal axes coincide with the axes of symmetry. In general, an orthogonal coordinate system is set up with their origin at the center of mass. When computed in the principal axis coordinate system, the inertia matrix is diagonal [18].

The basic parameters that were used for registration of the kidney are the position of the center of mass, the rotation of the kidney about the center of mass, and the lengths of the principle axes. These properties uniquely determine the location and geometry of the kidney in the three-dimensional space. After overlaying these 10 registered volumes, a probability model was created for each voxel based on how many kidney models are labeled as kidney tissue at that region. Figure 4 shows the overlaying of 10 segmented kidneys before and after registration. 


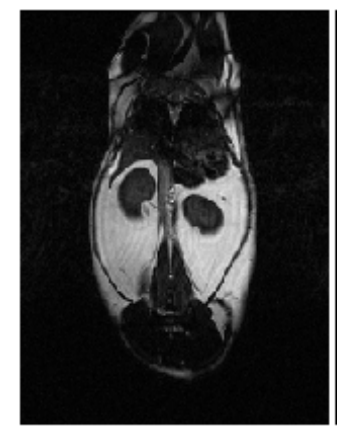

(a)

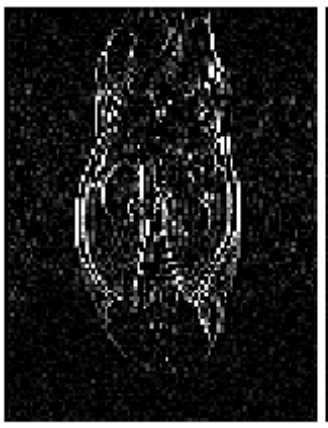

(b)

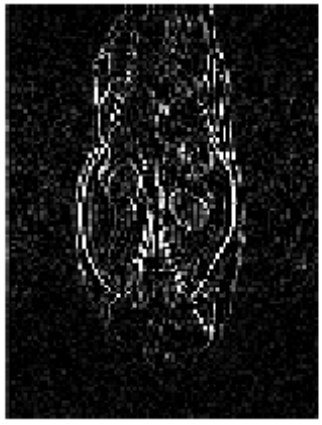

(e)

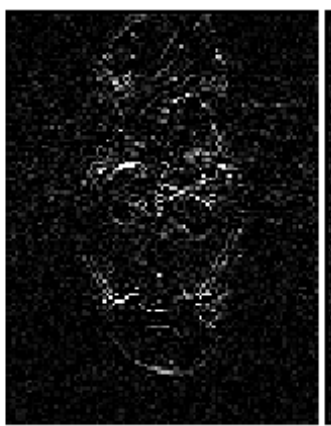

(c)

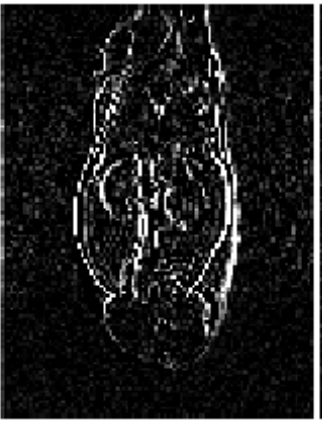

(f)

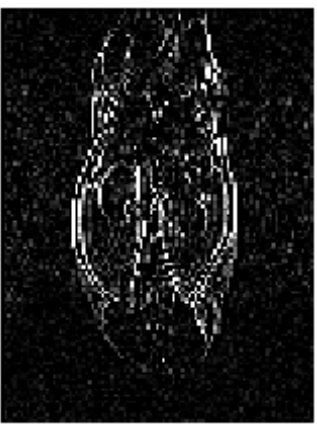

(d)

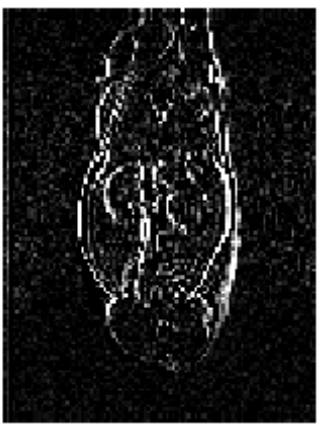

$(\mathrm{g})$

Figure 3. Wavelet filters that were employed for feature extraction: (a) Original image, (b) Daubechies 2 vertical details, (c) Symlets 2 horizontal details, (d) Symlets 2 vertical details, (e) Coiflets 1 vertical details, (f) Biorthogonal 1.3 vertical details, (g) Biorthogonal 1.5 vertical details.
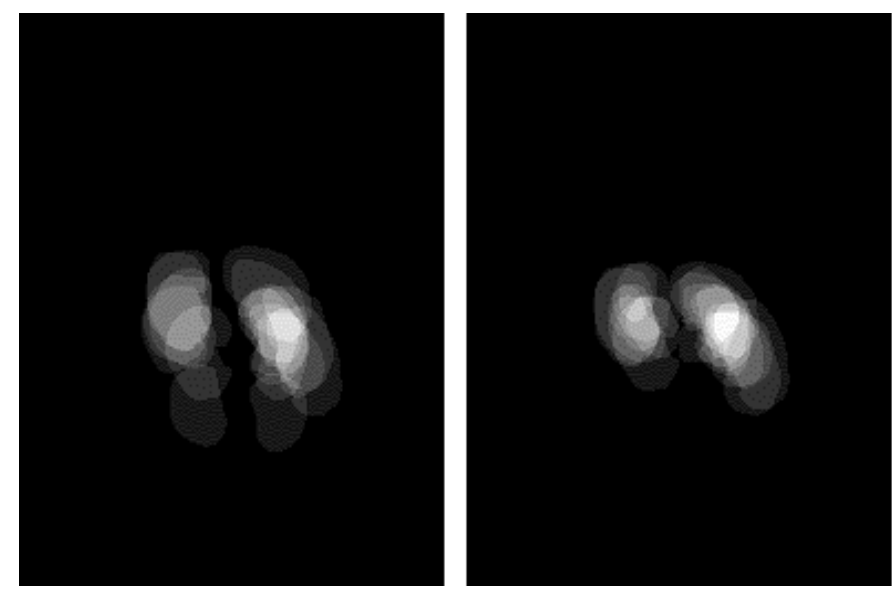

Figure 4. A section of the 3D probability model. The white voxels indicate that all segmented models are the kidney voxel. The black indicates that all segmented models are non-kidney voxels. The voxels in gray shows the probabilities based on their intensities. Left panel shows the model before registration and right panel shows the model after registration. 


\subsection{Edge detection}

To detect the edge of the kidney, a 3D edge detection method was defined. It is based on the Canny edge detection method. This method can detect the edge even in noisy images as shown in Figure 5. This edge is used for localizing the model. The highest intensity regions in the MR images are fatty tissue or some regions around the kidney. Therefore, this image was added to the detected edge to define the kidney edge at the lower location.

\subsection{Evaluation}

Quantitative performance assessment of the method was done by comparing the results with the corresponding gold standard data from manual segmentation. The Dice similarity was used as performance assessment metric in the kidney segmentation algorithm. The Dice similarity was computed as follows [19-21]:

$$
\operatorname{Dice}(S, G)=\frac{2|S \cap G|}{|S|+|G|} \times 100 \%,
$$

where $S$ represents the voxel set of the kidney segmented by the algorithm and $G$ represents the voxels of the kidney from the gold standard data.

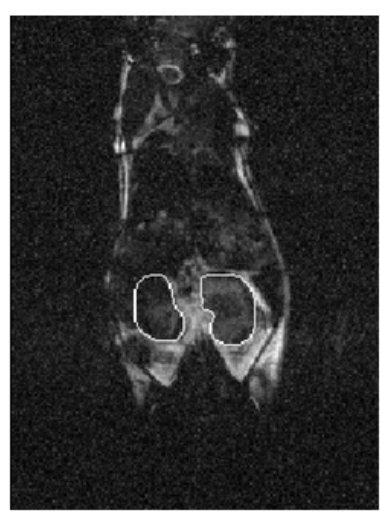

(a)

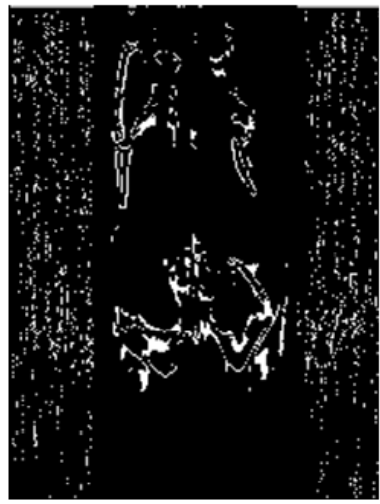

(d)

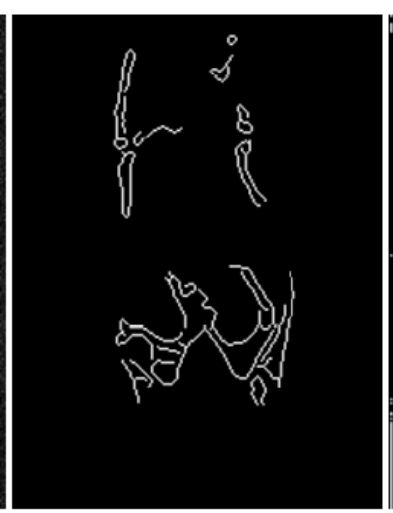

(b)

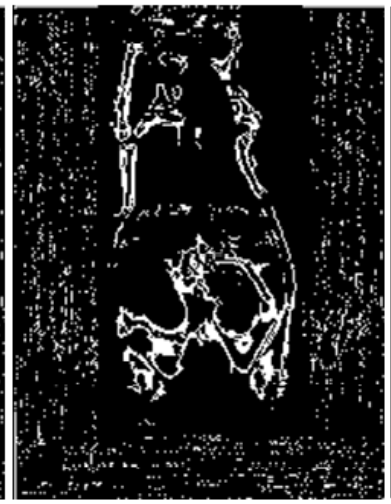

(e)

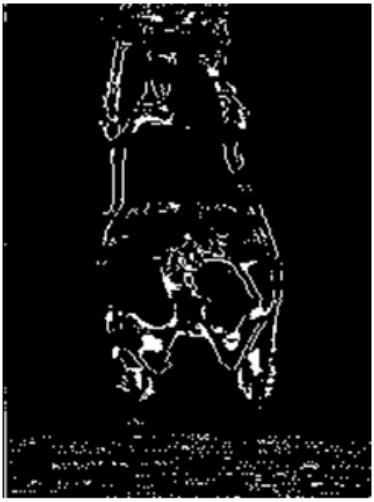

(c)

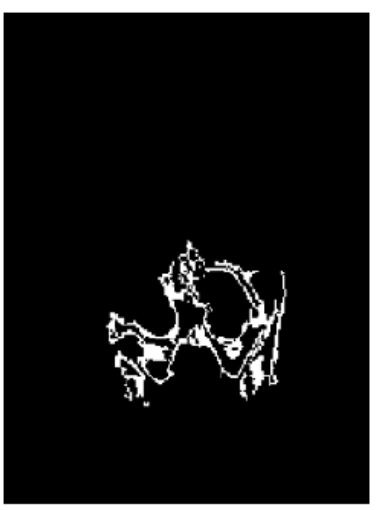

(f)

Figure 5. An example of the edge detection results: (a) Original image with the gold standard showed with white lines, (b) Coronal 2-D edge detection, (c) Transverse 2-D edge detection, (d) Sagittal 2-D edge detection, (e) 3D edge detection, (f) The 3D edge. 


\section{RESULTS}

The method was evaluated by MR data sets from seven mice, which were different from the MR data that were used for making model and training. Figure 6 shows an example of segmentation and its comparison with the corresponding gold standard. The numerical result of the Dice is shown in Table 1.

Table 1. Quantitative evaluation results.

\begin{tabular}{|c|c|c|c|c|c|c|c|c|c|}
\hline & $\begin{array}{c}\text { Mouse } \\
1\end{array}$ & $\begin{array}{c}\text { Mouse } \\
2\end{array}$ & $\begin{array}{c}\text { Mouse } \\
\mathbf{3}\end{array}$ & $\begin{array}{c}\text { Mouse } \\
4\end{array}$ & $\begin{array}{c}\text { Mouse } \\
5\end{array}$ & $\begin{array}{c}\text { Mouse } \\
6\end{array}$ & $\begin{array}{c}\text { Mouse } \\
7\end{array}$ & Mean & Standard Deviation \\
\hline DICE \% & 89.8 & 90.5 & 89.3 & 90.2 & 92.4 & 90.2 & 92.0 & 90.6 & 1.2 \\
\hline
\end{tabular}

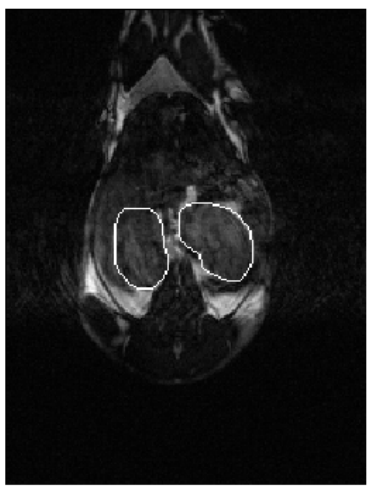

(a)

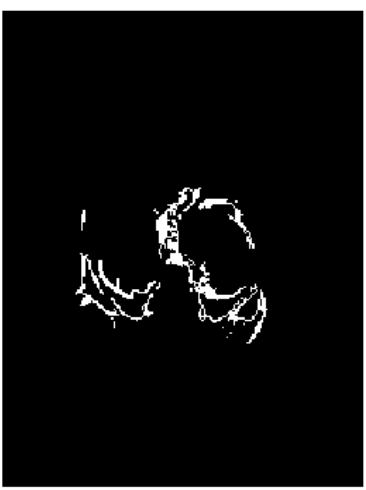

(b)

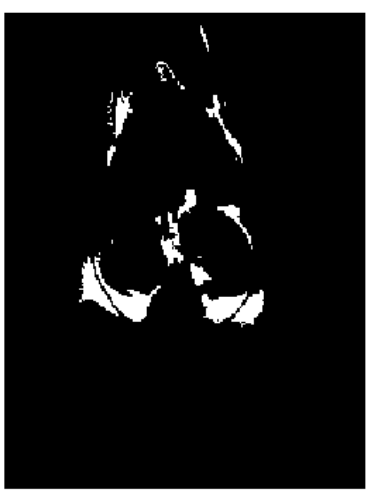

(c)

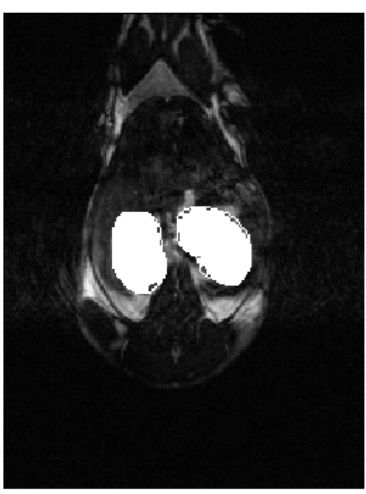

(d)

Figure 6. Segmentation results. (a) Original image with the white lines showing the gold standard boundaries, (b) 3D edge detection, (c) Fatty tissue detection, (d) The segmented result.

\section{DISCUSSION AND CONCLUSION}

A set of Wavelet-based support vector machines (W-SVMs) and a shape model were developed and evaluated for automatic segmentation of the kidney MR images. Wavelet transform was employed for kidney texture extraction. The segmentation results were incorporate with a probability kidney model to find a robust method for kidney segmentation. A set of W-SVMs are located on different regions of the kidney to classify kidney and non-kidney tissues in different zones around the kidney boundary. The method employs a learning based mechanism, using W-SVMs, to automatically collect texture features in different regions of the kidney. The probability model was incorporated into the segmented kidney to adaptively identify kidney and non-kidney tissues. In this way, even if the kidney has diverse appearance at different parts and has weak boundaries near liver, pancreas, or spleen, the model is still able to produce a relatively accurate segmentation in 3D MR images.

\section{ACKNOWLEDGEMENT}

This research is supported in part by NIH grant R01CA156775 (PI: Fei), Coulter Translational Research Grant (PIs: Fei and $\mathrm{Hu}$ ), Georgia Cancer Coalition Distinguished Clinicians and Scientists Award (PI: Fei), Emory Molecular and Translational Imaging Center (NIH P50CA128301), and Atlanta Clinical and Translational Science Institute (ACTSI) that is supported by the PHS Grant UL1 RR025008 from the Clinical and Translational Science Award program. 


\section{REFERENCES}

[1] V. E. Torres, and P. C. Harris, “Autosomal dominant polycystic kidney disease,” Nefrologia, 23, 14-22 (2003).

[2] P. Igarashi, and S. Somlo, "Genetics and pathogenesis of polycystic kidney disease," Journal of the American Society of Nephrology, 13(9), 2384-2398 (2002).

[3] P. D. Wilson, "Mechanisms of disease: Polycystic kidney disease," New England Journal of Medicine, 350(2), 151-164 (2004).

[4] M. Sutters, and G. G. Germino, "Autosomal dominant polycystic kidney disease: Molecular genetics and pathophysiology," Journal of Laboratory and Clinical Medicine, 141(2), 91-101 (2003).

[5] P. A. Gabow, A. M. Johnson, W. D. Kaehny et al., "Factors Affecting The Progression Of Renal-Disease In Autosomal-Dominant Polycystic Kidney-Disease," Kidney International, 41(5), 1311-1319 (1992).

[6] T. L. Chenevert, C. R. Meyer, B. A. Moffat et al., "Diffusion MRI: a new strategy for assessment of cancer therapeutic efficacy," Mol.Imaging, 1(4), 336-343 (2002).

[7] S. K. Lyons, “Advances in imaging mouse tumour models in vivo,” J.Pathol., 205(2), 194-205 (2005).

[8] K. Li, and B. Fei, "A New 3D Model-Based Minimal Path Segmentation Method For Kidney MR Images." The $2^{\text {nd }}$ International Conference on Bioinformatics and Biomedical Engineering - Proceedings of IEEE, 1, 23422344 (2008).

[9] K. Li, and B. Fei, "A Deformable Model-based Minimal Path Segmentation Method for Kidney MR Images." Proceedings of SPIE Medical Imaging: Imaging Processing, 6914, 69144F-1 7 (2008)

[10] B. Fei, C. Flask, H. Wang et al., "Image Segmentation, Registration and Visualization of Serial MR Images for Therapeutic Assessment of Polycystic Kidney Disease in Transgenic Mice.," Conf Proc IEEE Eng Med Biol Soc, 1, 467-9 (2005).

[11] H. Akbari, X. Yang, L. Halig et al., "3D Segmentation of Prostate Ultrasound Images using Wavelet Transform." Proceedings of SPIE Medical Imaging - Image Processing, 7962, 79622K-1 8 (2011)

[12] K. Bogie, X. Wang, B. Fei et al., "New technique for real-time interface pressure analysis: getting more out of large image data sets.," J Rehabil Res Dev, 45(4), 523-35 (2008).

[13] B. Fei, H. Wang, R. F. Muzic et al., "Deformable and rigid registration of MRI and microPET images for photodynamic therapy of cancer in mice.," Med Phys, 33(3), 753-60 (2006).

[14] B. Fei, S. Lee, D. Boll et al., "Image Registration and Fusion for Interventional MRI Guided Thermal Ablation of the Prostate Cancer." Medical Image Computing And Computer-Assisted Intervention (MICCAI 2003) Lecture Notes in Computer Science, 2879, 364-372 (2003)

[15] X. Chen, R. C. Gilkeson, and B. W. Fei, "Automatic 3D-to-2D registration for CT and dual-energy digital radiography for calcification detection," Medical Physics, 34(12), 4934-4943 (2007).

[16] B. W. Fei, J. L. Duerk, D. B. Sodee et al., "Semiautomatic nonrigid registration for the prostate and pelvic MR volumes," Academic Radiology, 12(7), 815-824 (2005).

[17] B. Fei, Z. Lee, J. L. Duerk et al., "Registration and Fusion of SPECT, High Resolution MRI, and interventional MRI for Thermal Ablation of the Prostate Cancer," IEEE Transactions on Nuclear Science, 51(1), 177-183 (2004).

[18] N. M. Alpert, J. F. Bradshaw, D. Kennedy et al., "The principal axes transformation--a method for image registration," J Nucl.Med, 31(10), 1717-1722 (1990).

[19] H. S. Wang, and B. W. Fei, "A modified fuzzy C-means classification method using a multiscale diffusion filtering scheme," Medical Image Analysis, 13(2), 193-202 (2009).

[20] X. F. Yang, and B. W. Fei, "A multiscale and multiblock fuzzy C-means classification method for brain MR images," Medical Physics, 38(6), 2879-2891 (2011).

[21] X. Yang, and B. Fei, "A skull segmentation method for brain MR images based on multiscale bilateral filtering scheme." Proceedings of SPIE Medical Imaging: Image Processing, 7623,76233K-1 8 (2010) 
Hamed Akbari and Baowei Fei, "Automatic 3D segmentation of the kidney in MR images using wavelet feature extraction and probability shape model", Proc. SPIE 8314, 83143D (2012)

Copyright 2012 Society of Photo-Optical Instrumentation Engineers (SPIE). One print or electronic copy may be made for personal use only. Systematic reproduction and distribution, duplication of any material in this paper for a fee or for commercial purposes, or modification of the content of the paper are prohibited.

http://dx.doi.org/10.1117/12.912028 